\title{
Folate receptor-targeted ultrasonic PFOB nanoparticles: Synthesis, characterization and application in tumor-targeted imaging
}

\author{
KESHI LI $^{1 *}$, YAHUI LIU ${ }^{1 *}$, SHENGMIN ZHANG ${ }^{1}$, YOUFENG XU ${ }^{1}$, JIANSHUAI JIANG $^{1}$, FENGYING YIN $^{1}$, \\ YUE HU ${ }^{1}$, BAOSAN HAN ${ }^{2}$, SHUXIONG GE $^{3}$, LI ZHANG $^{3}$ and YONG WANG ${ }^{1}$ \\ ${ }^{1}$ Department of Hepatobiliary Surgery, Ningbo First Hospital, Haishu, Ningbo, Zhejiang 315010; \\ ${ }^{2}$ Xinhua Hospital Affiliated to Shanghai Jiaotong University School of Medicine, Yangpu, Shanghai 200092; \\ ${ }^{3}$ Ningbo Medical School of Ningbo University, Jiangbei, Ningbo, Zhejiang 315211, P.R. China
}

Received February 23, 2016; Accepted April 24, 2017

DOI: $10.3892 /$ ijmm.2017.2975

\begin{abstract}
In this study, we aimed to determine an effective strategy for the synthesis of folate receptor (FR) targetednanoparticles (FRNPs). The nanoparticles used as ultrasound contrast agents (UCAs) were composed of a liquid core of perfluorooctyl bromide (PFOB) liposome and a targeted shell chemically conjugated with folic acid (FA) and polyethylene glycol (PEG). This was done in order to avoid recognition and clearance by the mononuclear phagocyte system [also known as the reticuloendothelial system (RES)] and enhance the targeting capability of the nanoparticles to tumors overexpressing folate receptor (FR). The FRNPs exhibited an average particle size of $301 \pm 10.8 \mathrm{~nm}$ and surface potential of $39.1 \pm 0.43 \mathrm{mV}$. Subsequently, in vitro, FRNPs labeled with FITC fluorescence dye were visibly uptaken into the cytoplasm of FR-overexpressing cancer cells (Bel7402 and SW620 cells), whereas the A549 cells expressing relatively low levels of FR just bound with few FRNPs. These results demonstrated that FRNPs have a high affinity to FR-overexpressing cancer cells. Additionally, in in vivo experiments, FRNPs achieved a greater enhancement of tumor ultrasound imaging and a longer enhancement time in FR-overexpressing tumors and the Cy7-labeled FRNPs exhibited a relatively high tumor-targeted distribution in FR-overexpressing tumors. Targeted ultrasound and fluorescence imaging revealed that FRNPs have the ability to target FR-overexpressing tumors and ex vivo fluorescence imaging was then used to further verify and confirm the pres-
\end{abstract}

Correspondence to: Dr Yong Wang, Department of Hepatobiliary Surgery, Ningbo First Hospital, 59 Liutin Street, Haishu, Zhejiang 315010, P.R. China

E-mail: robinwang9402@sina.com

${ }^{*}$ Contributed equally

Key words: ultrasound contrast agents, perfluorooctyl bromide, folate receptor-targeted nanoparticles, layer-by-layer assembly, tumor imaging ence of FRNPs in tumor tissues with histological analysis of the tumor slices. On the whole, our data demonstrate that the FRNPs may prove to be a promising candidate for the early diagnosis for FR-overexpressing tumors at the molecular and cellular levels.

\section{Introduction}

Ultrasound contrast agents (UCAs) are widely used in clinical practice in order to enhance the diagnostic capability and utility of traditional ultrasound imaging modes. The UCAs currently used commercially are usually designed to serve as blood pool agents with diameters of 1-8 $\mu \mathrm{m}$ and cannot pass through the blood vessel wall into the tumor tissue to achieve the molecular imaging of tumor tissue (1). Additionally the vascular endothelial gap in tumors is approximately $380-780 \mathrm{~nm}$, which is much wider than that of normal tissue $(2,3)$. In order to ensure that UCAs pass through the tumor tissue, researchers have paid more attention to the preparation of nanosized UCAs, due to their potential for the extravascular molecular imaging of tumors (4).

Although nanosized UCAs achieve passive imaging for tumors via enhanced permeability and retention (EPR), the specific capability of nanosized UCA to bind with tumor tissue is still limited (5). The technique which involves labeling nanoparticles with specific ligands can generate targeted nanoparticles capable of binding to specific tissues or tumors (6-8). Following intravenous injection, the nanoparticles aggregate specifically in the target tissues via the blood circulation, thus allowing the ultrasonography-based specific imaging of pathogenic changes at a molecular or cellular level (9).

Folate receptor (FR) binds folic acid (FA) with high affinity and mediates its intracellular transport via receptor-mediated endocytosis (10). There are three isoforms of FR (FR $\alpha, F R \beta$ and FR $\gamma$ ) and type $\alpha$ is the major isoform mediating folate transport, and is overexpressed on the surface of various types of tumor, including pancreatic, prostate, lung, head and neck, breast and ovarian cancer, and mesothelioma (11-19). Based on its limited expression and restricted distribution pattern in normal tissue, FR $\alpha$ is the most widely studied member of the 
FR family, and various strategies for targeting FR, which is overexpressed in cancer, have been developed $(20,21)$.

However, very few techniques involving ultrasound nanoparticles coupled with FR targeting and layer-by-layer (LbL) self-assembly have been described, to the best of our knowledge $(22,23)$. In the present study, we developed nanosized and highly efficient targeted nanoparticles modified with FA and polyethylene glycol (PEG) to verify the hypothesis that targeted nanoparticles can enhance the diagnostic value of ultrasound in FR-overexpressing tumors.

\section{Materials and methods}

Materials. N-Hydroxysuccinimide (NHS) were supplied by Sinopharm ChemicalReagent(Shanghai,China).Perfluorooctyl bromide (PFOB), FA and N-(3-Dimethylaminopropyl)-N'ethylcarbodiimide hydrochloride (EDC) were obtained from Sigma-Aldrich (St. Louis, MO, USA). PEG $\left(\mathrm{NH}_{2}-\mathrm{PEG}_{3400}\right)$ was obtained from Xibao Biochemicals (Shanghai, China). Chitosan (CS; degree of deacetylation 95\%) was obtained from Haidebei Marine Bioengineering (Shandong, China). Alginate (ALG) was obtained from Yuanye Biotech (Shanghai, China). Antifolate binding protein antibody (EPR4708) was obtained from Abcam (Cambridge, UK). Fluorescein isothiocyanate (FITC), DiI and immobilon-P transfer membrane were purchased from Solarbio (Beijing, China). Cy7 NHS ester (MW 720.94) was obtained from FANBO Biochemicals (Beijing, China). The PrimeScript $^{\mathrm{TM}}$ RT reagent kit with gDNA eraser and SYBR Premix Ex Taq were obtained from Takara (Shiga, Japan).

Cell culture and animal preparation. Bel7402 (human liver cancer cells), SW620 (human colorectal cancer cells) and A549 (human lung cancer cells) were purchased from the Cell Bank of Chinese Academy of Sciences (Shanghai, China). BALB/c 3T3 fibroblasts were purchased from the Cell Bank of Zhongshan University (Guangzhou, China). The cells were cultured in RPMI-1640 medium supplemented with $10 \%$ heat-inactivated fetal bovine serum at $37^{\circ} \mathrm{C}, 5 \% \mathrm{CO}_{2}$ cell incubator.

BALB/c nude male mice $(n=54 ; 5-6$ weeks old; body weight, 17-21 g; SLAC Laboratory Animals, Shanghai, China) kept in a specific pathogen-free environment were used as described below. All animal experiments were approved by the Animal Ethics Committee of the Medical School of Ningbo University (Ningbo, China) in accordance with the National Institutes of Health Guide for the Care and Use of Laboratory Animals and the ARRIVE guidelines. Under sterile conditions, logarithmic-phase Bel7402, SW620 (FR overexpressing cancer cells) and A549 (FR underexpressing cancer cells) were used to prepare cell suspensions at a density of $1 \times 10^{7}$ cells $/ \mathrm{ml}$; BALB/c 3T3 fibroblasts in the logarithmic phase growth were used prepare cell suspensions of $5 \times 10^{6}$ cells $/ \mathrm{ml}$. The mixture containing each type of cancer cell suspension and 3T3 fibroblast suspension (9:1 volume radio, $100 \mu$ l each mouse) was subcutaneously inoculated into the 5-6-week-old male BALB/c nude mice. Twenty animals were used for each xenograft tumor type. The xenografts were monitored daily with a venire caliper until the subcutaneous tumors reached $0.5-1.0 \mathrm{~cm}$ in diameter for 16-31 days after implantation.
Detection of FR expression in 3 types of cancer cells and xenografts

FR $m R N A$ expression in cancer cell lines detected by reverse transcription-quantitative PCR (RT-qPCR). RT-qPCR was used to measure the mRNa expression of FR $\alpha$ in the 3 cancer cell lines. Total RNA was extracted from the 3 types of cancer cells using TRIzol reagent protocol (Invitrogen, Carlsbad, CA, USA). Reverse transcription was performed using $500 \mathrm{ng}$ of total RNA and the PrimeScript ${ }^{\mathrm{TM}}$ RT reagent kit according to the manufacturer's instructions, and PCR was performed using the following primers: human FR $\alpha$ (sense strand, 5'-AGGTGCCATCTCTCCACAGT-3' and antisense, 5'-GAGGACAAGTTGCATGAGCA-3'; cDNA amplicon size: $135 \mathrm{bp}$; Tm, $60^{\circ} \mathrm{C}$ ); human GAPDH (sense, 5'-TTAAAAGCAGCCCTGGTGAC-3' and antisense, 5'-CTCTGCTCCTCCTGTTCGAC-3'; cDNA amplicon size: $\left.138 \mathrm{bp} ; \mathrm{Tm}, 60^{\circ} \mathrm{C}\right)$. cDNA was measured by using SYBR-Green RT-PCR in a StepOnePlus Real-Time PCR system (Thermo Fisher Scientific, Inc., Waltham, MA, USA) at $95^{\circ} \mathrm{C}$ for $30 \mathrm{sec}$, 40 cycles of $95^{\circ} \mathrm{C}$ for $5 \mathrm{sec}$ and $60^{\circ} \mathrm{C}$ for $34 \mathrm{sec}$. All PCR reactions were performed at least in triplicate and the mRNA levels were represented as relative quantification, which was calculated using relative expression of FR $(\mathrm{NFR} \alpha)=2^{-\Delta \Delta \mathrm{Cq}}$, where $\mathrm{Cq}=\Delta \mathrm{Cq}$ sample $-\Delta \mathrm{Cq}$ calibrator $(\Delta \mathrm{Cq}=\mathrm{Cq}$ of target gene - $\mathrm{Cq}$ of GAPDH).

Western blot analysis and immunohistochemical staining of FR in tumor tissues. Each tumor xenograt tissue (300 mg) was lysed on ice for $30 \mathrm{~min}$ in $1 \mathrm{ml}$ radioimmunoprecipitation assay protein lysis buffer (RIPA) followed by the addition of $10 \mu \mathrm{l}$ PMSF. All tumor tissue lysates were then transferred into a $1.5 \mathrm{ml}$ tube and centrifuged for $15 \mathrm{~min}$ at $15,000 \mathrm{rpm}$ at $4^{\circ} \mathrm{C}$. The supernatant was transferred to a new $1.5 \mathrm{ml}$ centrifuge tube. A bicinchoninicacid (BCA) kit (ComWin Biotech, Beijing, China) was then used to determine the protein concentration. Additionally, the samples were supplemented with 5X sodium dodecyl-polyacrylamide gel electrophoresis (SDS-PAGE) loading buffer, mixed and boiled for $5 \mathrm{~min}$. Twenty micrograms of total protein were separated by SDS-PAGE and transferred onto a polyvinylidene fluoride (PVDF) membrane. The membrane was blocked with $5 \%$ albumin bovine in TBST at room temperature for $1 \mathrm{~h}$. Subsequently the blots were incubated with $1: 5,000$ dilution $(0.145 \mu \mathrm{g} / \mathrm{ml})$ anti-folate binding protein monoclonal antibody (EPR4708; ab125030; Abcam) at $4^{\circ} \mathrm{C}$ overnight, followed by the addition of 1:500 dilution of goat anti-rabbit IgG HRP-conjugated secondary antibody (CW0103S; ComWin Biotech) at room temperature for $2 \mathrm{~h}$; the membrane was washed 3 times with TBST after each antibody incubation.

To detect FR expression by immunohistochemical staining, each tumor-bearing nude mouse was anesthetized intraperitoneally with $1 \%$ sodium pentobarbital and sacrificed when the subcutaneous tumors reached the required volume. After that, the tumors were completely removed from the xenografts and frozen by liquid nitrogen immediately. The fresh frozen blocks of the removed tumors were dissected into serial sections of approximately $4-\mu$ m-thick using a freezing microtome (CM1860; Leica Microsystems, Wetzlar, Germany). Each section was covered on a clean glass slide and fixed by $4 \%$ polyformaldehyde for $10 \mathrm{~min}$. The slides were then washed in phosphate-buffered saline (PBS), and soaked in 3\% hydrogen peroxide methanol solution for $15 \mathrm{~min}$. Each slide was incubated 
with 5\% goat serum albumin for 20 min to block non-specific antigen sites, and rabbit anti-folate binding protein monoclonal antibody (EPR4708; ab125030; Abcam) was added. These slides were placed inside a humidified chamber for $30 \mathrm{~min}$ at room temperature, then washed with PBS buffer for $5 \mathrm{~min}$ and dried. Subsequently, the streptavidin-biotin complex immunocytochemistry protocol was used for staining and mild re-dyeing with hematoxylin. Subsequently, the slides were dehydrated, covered slipped with mounting, and observed under a microscope (NIB-100; Olympus, Tokyo, Japan). The results were considered as positive when claybank particles were observed in the cytoplasm, cell membrane or nucleus.

Preparation of FR-targeted nanoparticles (FRNPS) and blank nanoparticles (NPs). The FR-targeted PFOB (FRNPs) were prepared to combine FA- and PEG-modified targeted shells with a PFOB nanocore template using the $\mathrm{LbL}$ assembly technology. PFOB nanocore templates were prepared by thin-film hydration and the ultrasonic emulsification method, as previously described (24,25). Firstly, egg lecithin and cholesterol (2:1 M radio) were dissolved into an organic solvent mixture of chloroform. The organic solvent was removed from the organic mixture in arotary evaporator (Yarong, Shanghai, China) at $50^{\circ} \mathrm{C}$ under a vacuum. The resulting thin film $(100 \mathrm{mg})$ was hydrated by using purified water $(5 \mathrm{ml})$ and then alginate $(100 \mathrm{mg})$ was added to to the solution. Subsequently, the mixture was supplemented with PFOB $(220 \mu \mathrm{l})$ in a dropwise manner and sonicated at a certain ultrasound intensity to form the PFOB nanocore template. The resulting PFOB nanocore templates also served as blank NPs for use in subsequent experiments.

FA conjugation to $\mathrm{CS}$ and ALG modified with $\mathrm{NH}_{2}$-PEG were carried out to form crosslinking between amino and carboxyl groups in the presence of EDC and NHS, which served as targeted shells, as previously described (23). Briefly, the synthesis of FA-CS was as follows: FA (220.7 mg) dissolved in $10 \mathrm{ml}$ of DMSO was activated with EDC (287.6 mg) and NHS (172.6 mg) at room temperature for $4 \mathrm{~h}$, and the activated solution was then added in a dropwise manner to $20 \mathrm{ml}$ of $2 \%$ acetic acid aqueous solution containing CS (161 mg) and stirred for $20 \mathrm{~h}$ at room temperature in the dark. Also, the synthesis of PEG-ALG was as follows: ALG (176 mg) dissolved in $10 \mathrm{ml}$ of DMSO was activated with EDC (46 mg) and NHS (27.6 mg)at room temperature for $4 \mathrm{~h}$, then the activated solution was then added in a dropwise manner to $20 \mathrm{ml}$ of $2 \%$ acetic acid aqueous solution containing PEG (273.2 mg) and stirred for $20 \mathrm{~h}$ at room temperature in the dark. The reaction products was dialyzed using dialysis membrane (molecular weight cut-off of 8-10 kDa) to remove unreacted substance and lyophilized.

Polymer (FA-CS and PEG-ALG) and PFOB liposome solutions were prepared at a concentration of $10 \mathrm{mg} / \mathrm{ml}$ and $30 \mathrm{mg} /$ $\mathrm{ml}$. Subsequently, $1 \mathrm{ml}$ polymer (FA-CS) solution was added to the liposome dispersions and vortexed for $10 \mathrm{~min}$. The mixture was then sonicated for $5 \mathrm{~min}$ and kept aside for PEG-ALG polymer solution addition (26). The above steps were repeated 3 times and the resulting products were incubated with $2 \mathrm{ml}$ polymer (FA-CS) solution for $30 \mathrm{~min}$. The FRNPs were then obtained by centrifugation $\left(12,000 \times \mathrm{g}, 10 \mathrm{~min}, 4^{\circ} \mathrm{C}\right)$ and washed 3 times using purified water. The FRNPs was dispersed in $5 \% \mathrm{w} / \mathrm{v}$ mannitol aqueous solution by low-frequency ultra- sound and filtrated by $0.45 \mu \mathrm{m}$ membrane filtration to remove large impurities.

\section{Characterization of nanoparticles}

Particle size and zeta potential. Firstly the appearance of FRNP emulsions was observed using a light microscope (NIB-100; Olympus). Particle size, polydispersity index (PDI) and zeta potential were obtained using a Malvern Zetasizer Nano ZS (Malvern Instrument, Malvern, UK) and size measurements were performed at $20^{\circ} \mathrm{C}$. The results were expressed as the mean diameter and size distribution of FRNPs, blank NPs obtained from 3 measurements. The standard deviation of the size and the PDI were also given. Before each measurement, $200 \mu \mathrm{l}$ of nanoparticles suspension were diluted into $1 \mathrm{ml}$ of PBS.

Transmission electron microscopy (TEM). The morphological characterization and internal structure of FRNPs and blank NPs were observed using a transmission electron microscopy JEOL 2100 HR (Jeol, Tokyo, Japan). Suspensions of FRNPs and blank NPs were deposited on filter papers to blot off the excess solution and air-dried by using an incandescent lamp before observation. Images were acquired using a high-resolution camera, Advantage Orius 831 (Gatan, Pleasanton, CA, USA).

Targeted binding assay of nanoparticles in vitro. Logarithmic-phase Bel7402, SW620 and A549 cells were stained with DiI before use. The stained cells were then seeded on coverslips placed in 12-well cell plates at a density of $2 \times 10^{4}$ cells/well divided into 2 groups and cultured for $24 \mathrm{~h}$. FITC-labeled FRNPs (1x10 ${ }^{13}$ particles/ml, $\left.20 \mu \mathrm{l}\right)$ were added to the first group, which served as the targeted group. FITClabeled blank NPs $\left(1 \times 10^{13}\right.$ particles $\left./ \mathrm{ml}, 10 \mu \mathrm{l}\right)$ were added to the second group, which served as the control group. After the cells were incubated with the nanoparticles for $6 \mathrm{~h}$, the coverslips were washed 3 times with PBS, fixed with $4 \%$ paraformaldehyde and finally washed with PBS. Subsequently, the coverslips were mounted onto glass slides and observed under a fluorescence microscope (DMIRB; Leica Microsystems).

Ultrasound targeted imaging of FRNPs in vivo. Nine animals were used for each xenograft tumor type in ultrasound imaging. The tumor-bearing nude mice were anesthetized intraperitoneally with $1 \%$ sodium pentobarbital for imaging, and the surfaces of both the tumor and probe were covered with a 6-mm-thick coupling agent. A LA332 broadband linear ultrasound probe connected to an Mylab90 ultrasound system (Esaote, Genova, Italy) was used to perform B mode ultrasound imaging of the xenografts. Once the cross-section of a xenograft was fully observed, the probe was immobilized and the parameters of ultrasonography mode were set up (mechanical index 0.7; gain $80 \%$ ). Each type of test tumor-bearing nude mice were divided into 3 groups: group A received $200 \mu \mathrm{l} \mathrm{FRNPs,} \mathrm{group} \mathrm{B}$ received $200 \mu \mathrm{l}$ blank NPs and group C received $200 \mu \mathrm{l}$ Sonovue. The contrast agents were injected into the test animals via the tail vein. The ultrasound images of the xeongrafts were collected prior to the injection and at 1, 5, 10, 20, 40, 80, 120 and $160 \mathrm{~min}$ post-injection; the imaging date were collected to calculate the increased imaging intensity at different time-points.

Fluorescence imaging of FRNPs in vivo. To validate the targeting capability of FRNPs in the other routine and investigate the biodistribution of FRNPs, we labeled the shell of FRNPs with Cy7 fluorescent dye (27) and each xenograft was intravenously injected with $200 \mu \mathrm{l}$ Cy7-labeled FRNPs. Six animals were used 


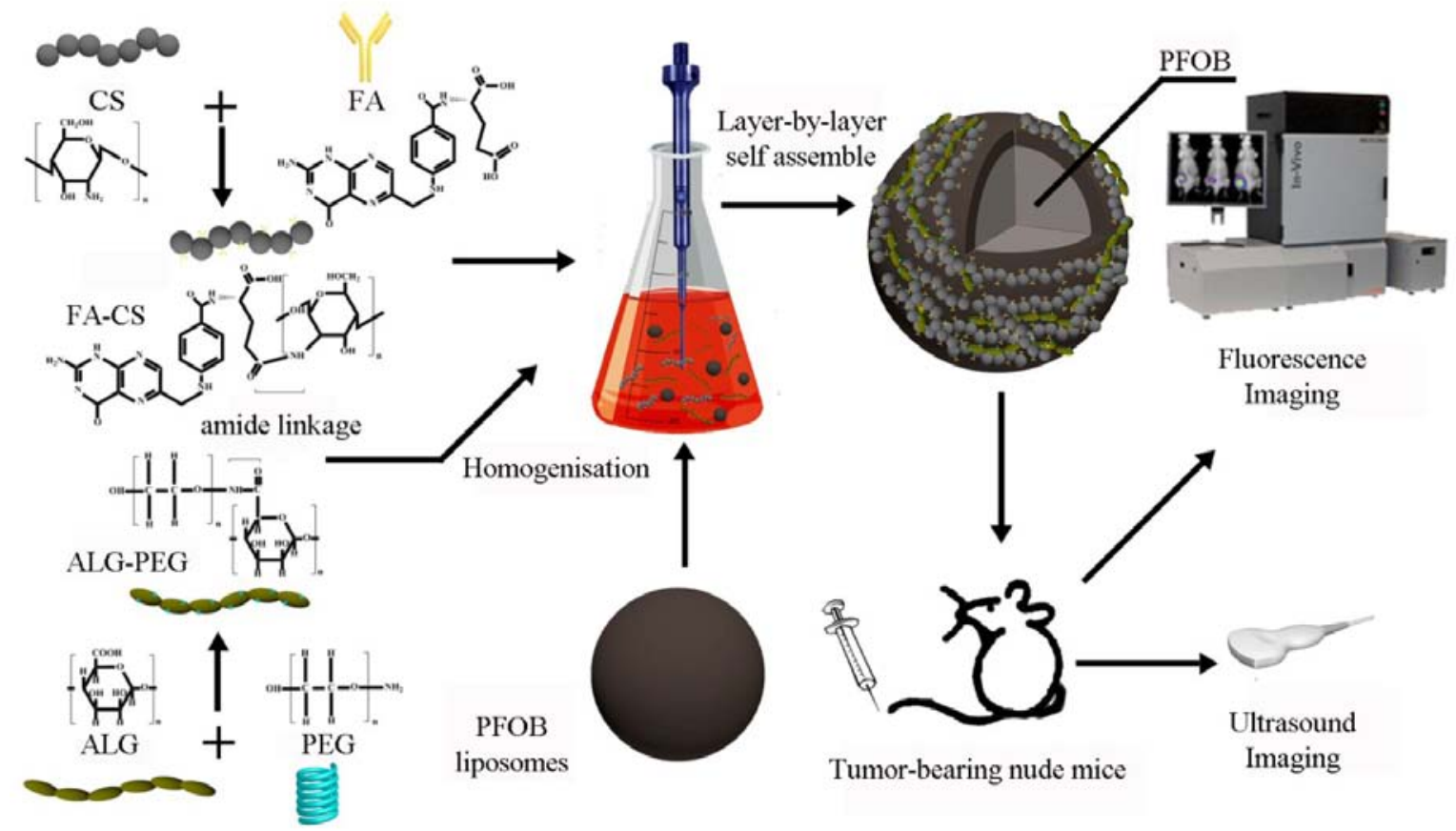

Figure 1. Schematic of the formulation process for folate-receptor targeted nanoparticles (FRNPs) and the nanoparticle in vivo fluorescence imaging and ultrasound imaging.

for each type of xenograft. Following administration by intravenous injection, the time-dependent biodistribution of the FRNPs in the xenografts was imaged using the Kodak In-vivo Imaging System FX Pro (excitation, $610 \mathrm{~nm}$; emission, $790 \mathrm{~nm}$; exposure time, $10 \mathrm{sec}$; Carestream Health, Rochester, NY, USA). The fluorescence images were then analyzed based on their spectral patterns using Carestream Molecular Imaging software. Scans were performed at 5, $30 \mathrm{~min}, 1,1.5,3$ and $12 \mathrm{~h}$ post-injection, after which the tumor-bearing mices were sacrificed. The tumors, heart, liver, spleen, lungs, kidneys and intestines were harvested and imaged to estimate the tissue distribution of FRNPs. To compare the targeting ability of FRNPs in different tumors, the target-to-background ratio (TBR) at different time points was obtained using the Carestream MI software to calculate regions of interest (ROI) functions. The ROIs were selected manually by drawing regions on the in vivo fluorescent images. The TBR was calculated using the following formula: $\mathrm{TBR}=\mathrm{SI}_{\mathrm{T}} / \mathrm{SI}_{\mathrm{M}}$, where $\mathrm{SI}_{\mathrm{T}}$ is the average signal intensity of the tumor and $\mathrm{SI}_{\mathrm{M}}$ is the average signal intensity of the contra lateral thigh muscle. This procedure was carried out as previously described by Alencar et al (28).

Histological analysis. Twelve animals (each type of xenografts taken from 4 mice) were divided into 2 groups, including the targeted group and the control group. The targeted group was intravenously injected with $200 \mu$ l Cy7-labeled FRNPs, whereas the control group received $200 \mu$ l physiological saline. At $2 \mathrm{~h}$ post-injection, the mice were sacrificed. Tumor tissues were carefully removed and fixed with $4 \%$ paraformaldehyde at $\mathrm{pH} 7.4$ for 4 days. The tumors were then washed with PBS, then embeded with optimal cutting temperature (OCT) and plalced into liquid nitrogen for storage. Frozen tissue blocks were dissected into $4-\mu \mathrm{m}$-thick sections and placed on clean glass slides. The slides were dehydrated, coverslipped and mounted, and examined under a fluorescence microscope (DMIRB; Leica Microsystems). Red fluorescence spots were observed with a long-pass 515-560 nm emission filter.

Statistical analysis. The experimental data were analyzed using Statistical Package for Social Science (SPSS) 19.0 software (SPSS Inc., Chicago, IL, USA). All the quantitative data were expressed as the means \pm standard deviation. The FRNPs and blank NB ultrasound indicator data from in vivo imaging were obtained and analyzed using Image-Pro Plus 6.0 software (Media Cybernetics Inc., Rockville, MD, USA). The ultrasound indicators of the nanoparticles in the 3 types of xenografts were analyzed by analysis of variance (ANOVA). A P-value $<0.05$ was considered to indicate a statistically significant difference. Histograms and the curve with non-linear regression were plotted using GraphPad Prism 5.0 software (GraphPad Software, La Jolla, CA, USA).

\section{Results}

Synthesis of the nanoparticles. In this study, we used PFOB as the core of the contrast agents, which is a classical, highly biocompatible alternative for nanosized ultrasound contrast agents. The synthesis of PEG-ALG and FA-CS was conducted using the carbodiimide method. Furthermore, we attempted to develop FR-targeted PFOB nanoparticles enveloped in a targeted shell consisting of PEG-ALG and FA-CS and explored the related characteristics, targeting ability, biodistribution in vivo and ultrasound contrast-enhancing potential and behavior. The process of LbL self-assembly is shown in Fig. 1.

Particle size and zeta potential characterization. The data of dynamic light scattering (DLS) analysis indicated that the alternate polymer assembly was accompanied by a gradual 


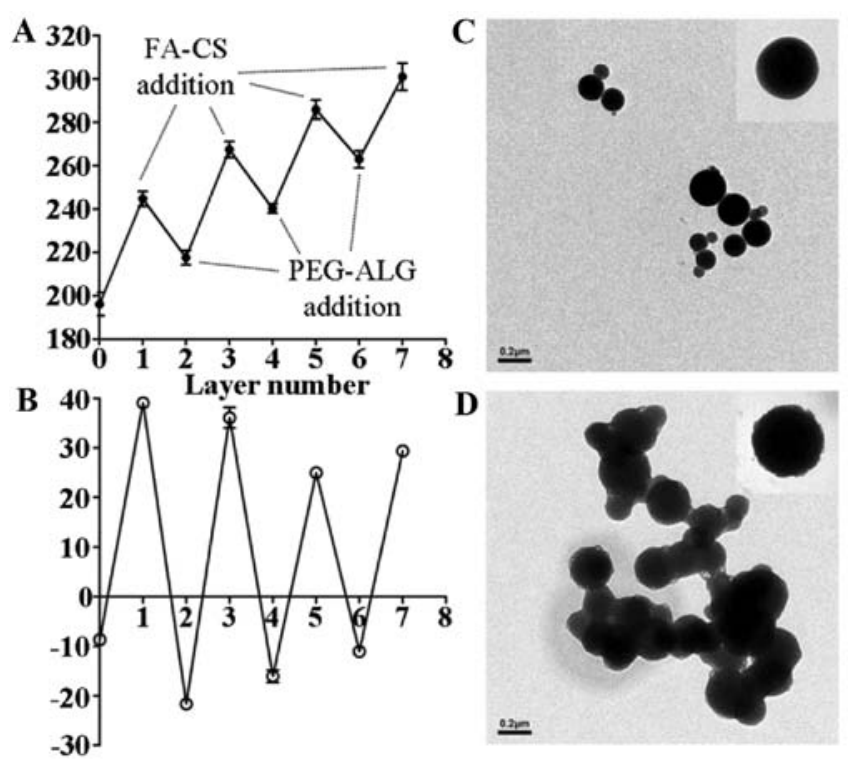

Figure 2. Variation trend of particle size (A); zeta potential with the addition of each polymer layer (B); transmission electron microscopy (TEM) images of blank NPs (C); TEM images of FRNP (D).

increase in particle size (Fig. 2A). Prior to LbL fabrication, blank NPs (PFOB liposomes) exhibited an average particle size of $196.2 \pm 9.3 \mathrm{~nm}$. In the LbL assembly process, the overall size of the nanoparticles decreased after each addition of PEG-ALG. This indicated that the strong ionic electrostatic interaction between PEG-ALG and FA-CS contributed to the formation of a mesh shell on the surface of PFOB liposome. After the process was completed, the final size of the FRNPs reached an average particle size of $301 \pm 10.8 \mathrm{~nm}$. Deposition of alternate polymers was accompanied by a reversal of surface potential (Fig. 2B), which indicated that a solid monolayer had been deposited. An abrupt rise in the liposome surface potential (from $-8.66 \pm 0.68$ to $39.1 \pm 0.43 \mathrm{mV}$ ) was observed after addition of the first (FA-CS) layer. However, the similar surface potential was restored upon addition of the second (PEG-ALG) layer.

Morphological analysis. TEM was used to observe the morphology and structure of the FRNPs directly. The blank NPs exhibited well-defined spherical shape with a smooth surface and small size (Fig. 2C). The FRNPs exhibited a rough surface and increased average diameter after the addition of polymers by LbL assembly (Fig. 2D): a darker phosphotungstic acid-stained core and a lesser-stained shell were visible. This indicated that the addition of polymer layers resulted in the formation of compact core-shell NPs.

Estabslishment of tumor xenograft animal model. Firstly, RT-qPCR was used to examine FR expression in the Bel7402, SW620 and A549 cancer cells. The results presented in Fig. 3A showed that among these 3 types of cancer cells,the Bel7402 cells expressed the highest level of FR, followed by the SW620 cells. The A549 cells exhibited the lowest expression level of FR. Subsequently, we developed certain types of tumor xenografts with these cancer cell lines by injecting the cell suspesions into mice and determined FR expression in the tumor tissue of these
3 types of xenografts (for each type of xenograft, we randomly selected 3 animals).

The results of western blot analysis (Fig. 3B) and immunohistochemical staining (Fig. 3C) of the tumor tissues were consistent with the results of RT-qPCR for the FR mRNA level in the 3 cancer cell lines. FR expression in the Bel7402 and SW620 tumor xenografts was much higher than that in the A549 tumor xenografts. This indicated that the animal models were successfully created and that the xenografts conformed to the quirement for the experiments of ultrasound targeted imaging and biodistribution in vivo.

Uptake of nanoparticles by the cancer cells. Since the FITClabeled FRNPs and blank NPs were developed to bind to FR and trigger receptor-mediated internalization in tumor cells, we compared the uptake of the targeted group and the control group in the Bel7402 cells, SW620 cells and A549 cells lines (Fig. 4). In the targeted group, a large number of green fluorescent spots representing the FRNPs were absorbed into the cytoplasm of Bel7402 and SW620 cells, whereas few green spots remained on the cell membrane of the A549 cells. Furthermore, in the control group, few green fluorescent spots representing the blank NPs were also observed on the 3 types of cell membrane. These results demonstrated that the FRNPs had a greater affinity to the Bel7402 cells and SW620 cells, in which FR was overexpressed.

\section{Ultrasound targeted imaging in vivo}

The peak enhancement of tumor ultrasound imaging results. The peak enhancement of tumor imaging is shown in Fig. 5. The ultrasonographic images among the 3 types of xenografts revealed that the enhancement of Sonovue was higher than the enhancement achieved with the FRNPs and blank NPs. However, the enhancement of the FRNPs in both the Bel7402 and SW620 tumor xenografts was apparently higher than the enhancement achieved with the blank NPs at the peak level. In the A549 tumor xenografts, the imaging results of the FRNPs and blank NPs were comparable at the peak level of enhancement. Thus, the blank NPs achieved the worst performance on tumor-enhanced imaging, and both the FRNPs and Sonovue obtained excellent tumor-enhanced imaging for FR overexpressing tumors.

Variation trend in the intensity value of tumor-enhanced imaging. Imaging Pro Plus 6.0 software was used to analyze the grey value of the ultrasound images. The difference in the grey valve of images collected at pre-injection and post-injection served as the intensity value of tumor imaging by 3 types of contrast agents in the xenografts. The time-intensity curve is shown in Fig. 6, illustrating the change in ultrasonic enhancement in areas of interest (AOI) of the tumors collected from the xenografts. From 1 to 10 min post-injection, the imaging of the tumors was significantly enhanced in the Sonovue group, and the intensity value of tumor imaging in this group was considerably higher than that of the other groups $(\mathrm{P}<0.001)$, with no clear differences among the 3 types of xenografts $(\mathrm{P}=0.996)$. At 20 min post-injection, the intensity value in the Sonovue group decreased rapidly, and almost disappeared within 40 min post-injection. However, the intensity value in the FRNPs and blank NP groups gradually increased after the injection and reached the peak levels at 10 and 20 min post-injection. In the 

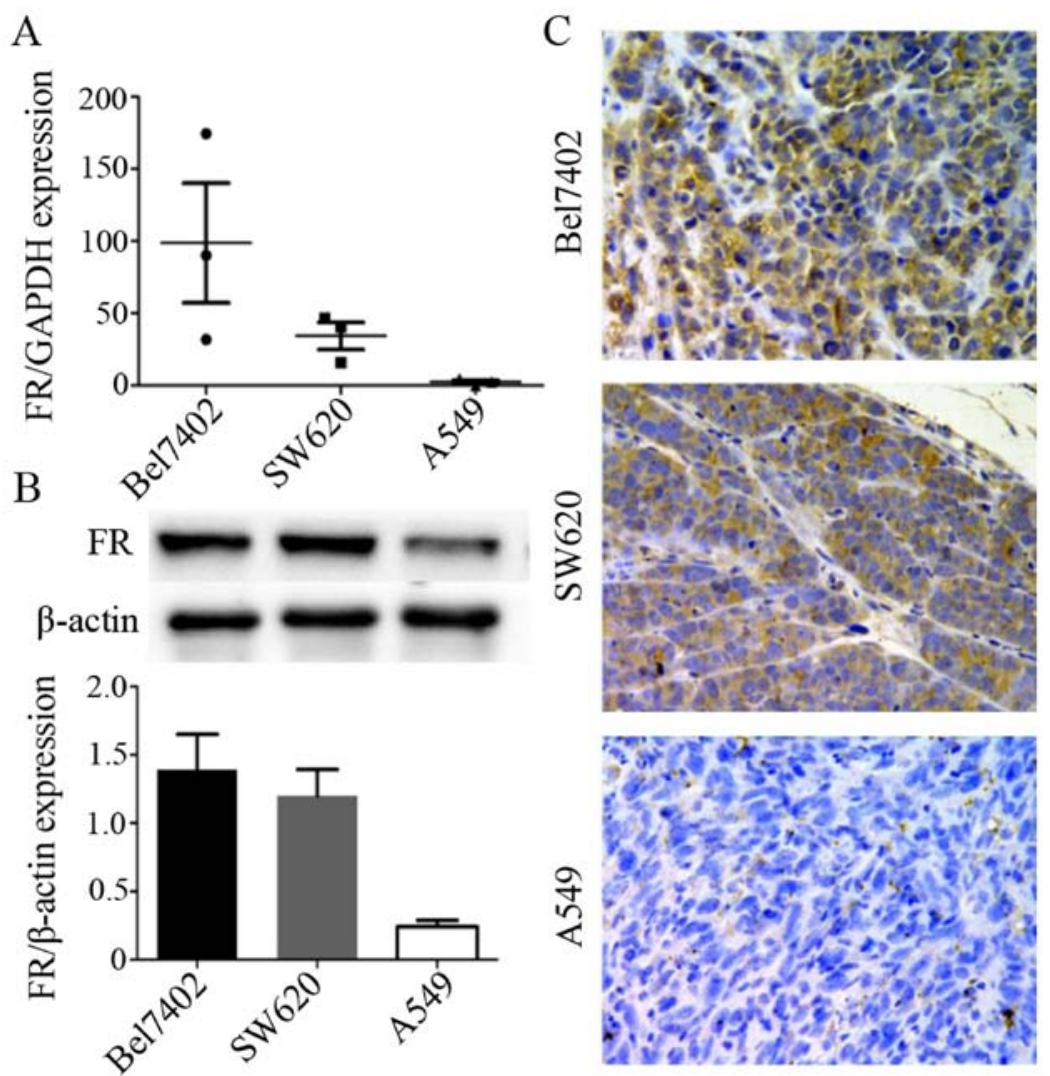

Figure 3. Folate receptor (FR) expression in the 3 cell lines was detected by RT-qPCR (A) and the expression in the tumor tissues of the corresponding xenografts detected by western blot analysis (B) and immunohistochemical staining (C). The tumor tissues of Bel7402 and SW620 tumor xenografts expressed higher levels of FR than those of the A549 tumor xenografts, and the differences in the data were considered statistically significant $(\mathrm{P}<0.05)$.
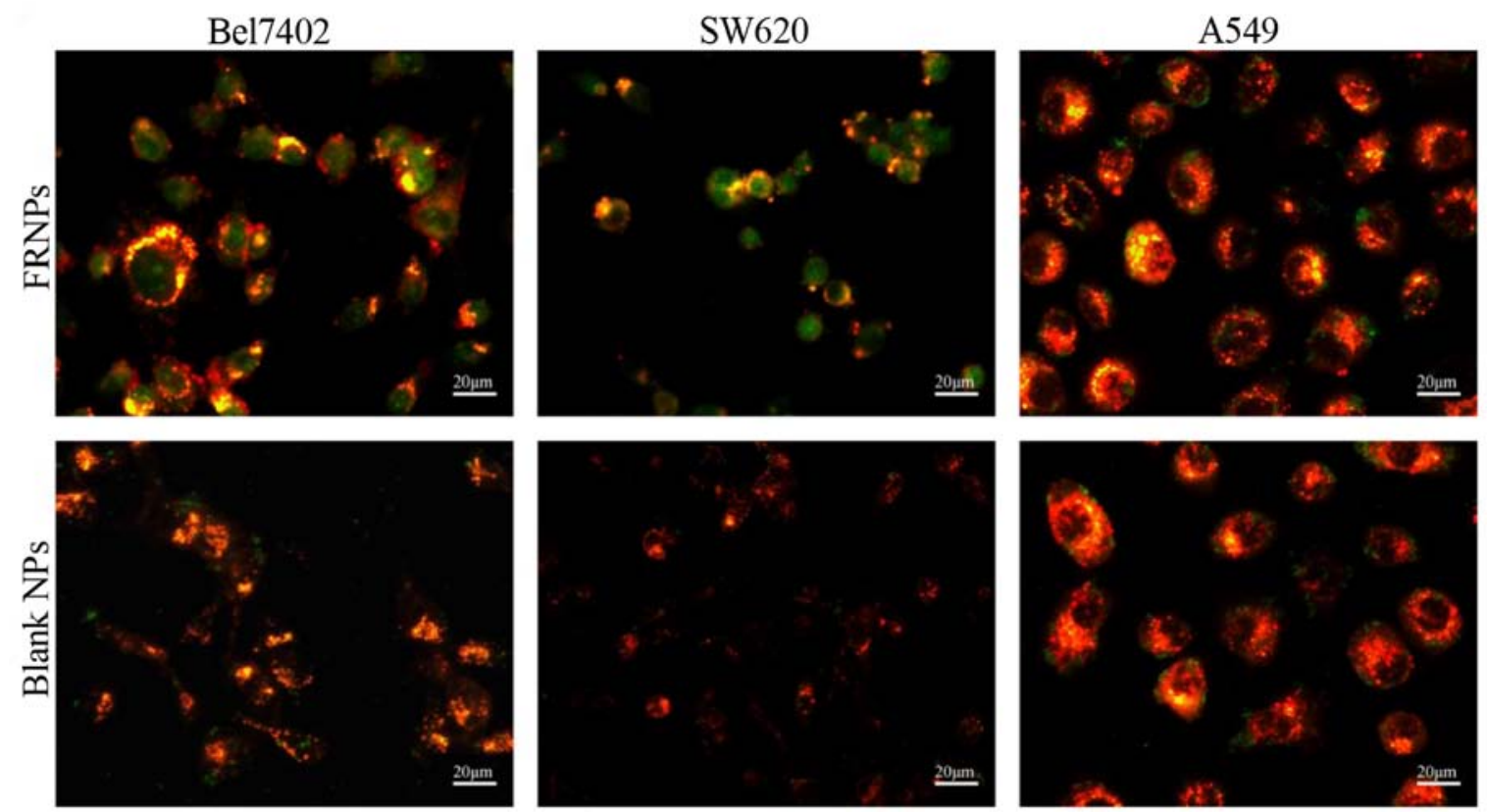

Figure 4. In vitro fluorescein isothiocyanate (FITC)-labeled folate-receptor targeted nanoparticles (FRNPs) and blank NPs (green dots) binding with 3 types of cancer cells (red area) were observed under a fluorescence microscope. FRNPs were visibly uptaken into the cytoplasm of both Bel7402 cells and SW620 cells, whereas A549 cells just bound with few FRNPs. None of the 3 types of cells displayed prominent binding to blank NPs.

FRNP group, the intensity value of the Bel7402 and SW620 tumor xenografts was significantly higher than that of the A549 tumor xenografts from 20 to 160 min post-injection $(\mathrm{P}=0.012)$.
Until the time point of $160 \mathrm{~min}$ post-injection, the enhancement of the FRNPs in the Bel7402 tumor xenografts and SW620 tumor xenografts remained at a high level of intensity, and this 
FRNPs
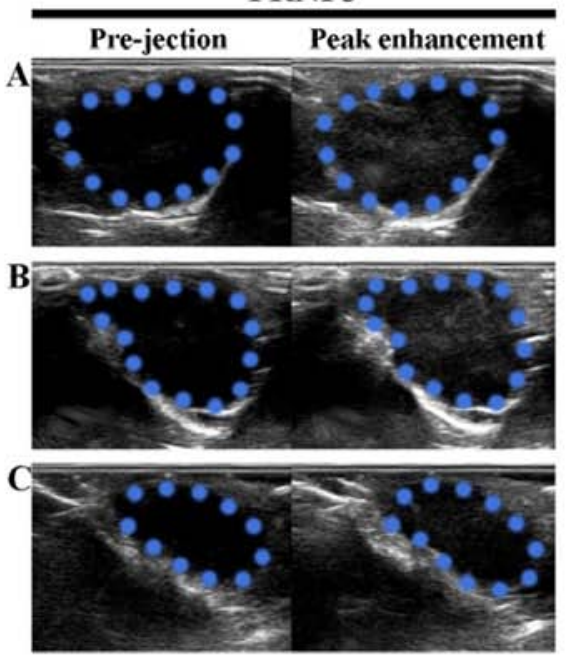

Blank NPs
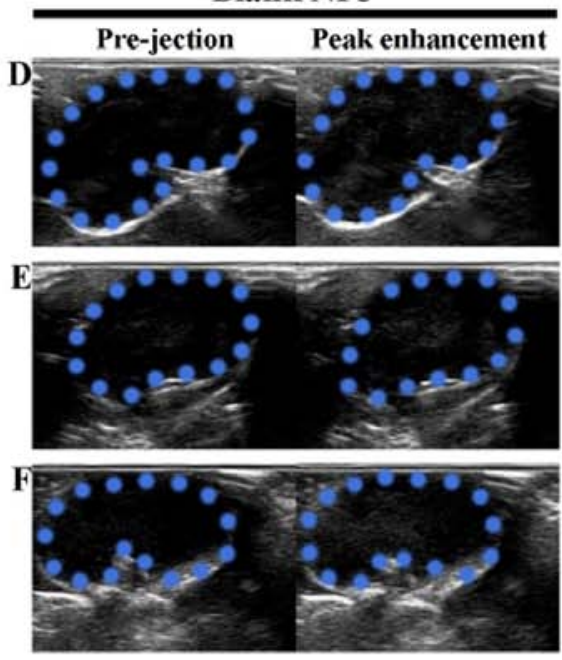

Sonovue
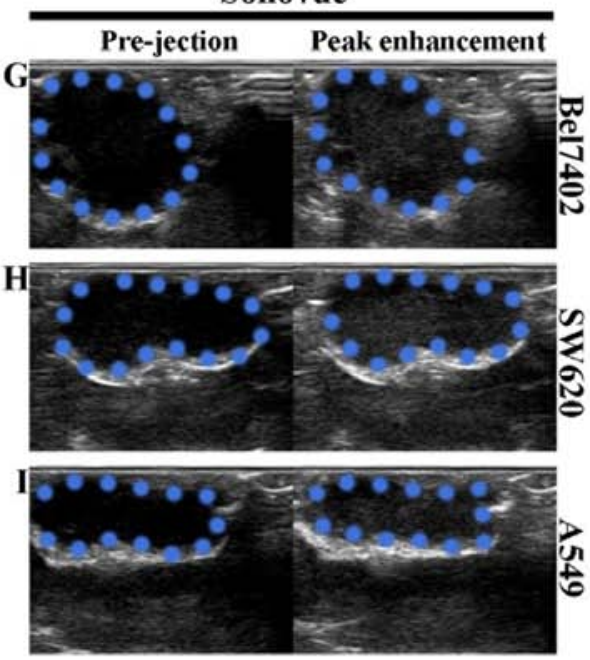

Figure 5. Imaging results of folate-receptor targeted nanoparticles (FRNPs), blank NPs and Sonovue in the 3 types of xenografts before injection and at the peak enhancement. (A, D and G) Changes in AOI after injection of FRNPs to the 3 types of xenografts under B-mode ultrasonography. (B, E and H) Corresponding changes with the injection of blank NPs. (C, F and I) Corresponding changes with injection of Sonovue. In all images, the areas circled by blue dotted lines represent the areas of interest (AOI) of tumor xenografts.

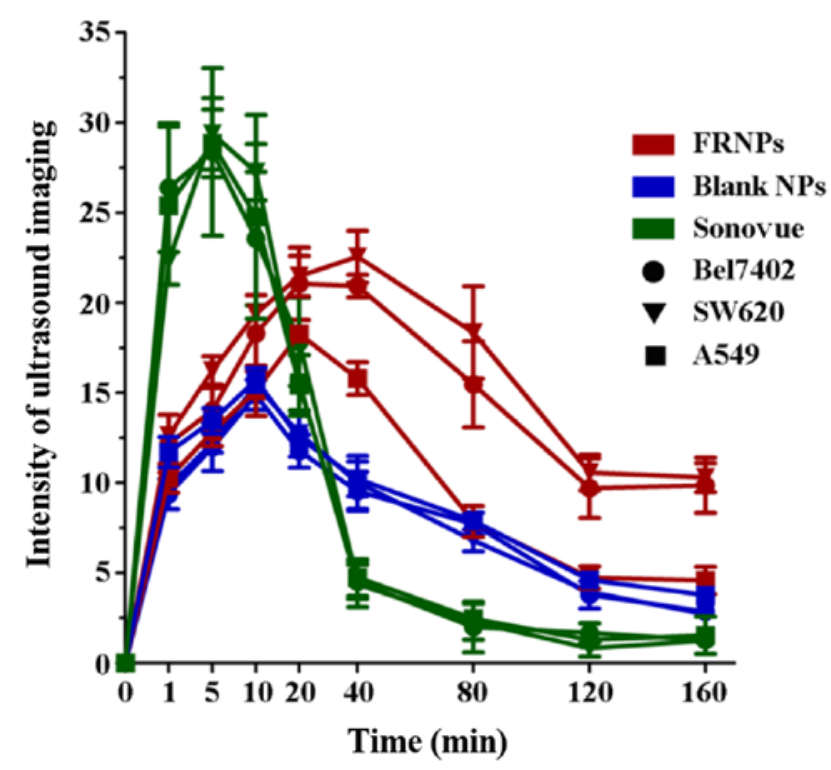

Figure 6. The time-intensity curve of folate-receptor targeted nanoparticles (FRNPs), blank NPs and Sonovue in the 3 types of xenografts. Red curves show the changes of enhancement after injection of FRNPs in xenografts, blue curves show the changes of enhancement after injection of NPs and green curves show the changes of enhancement after injection of Sonovue, whereas the circles represent the imaging data collect from Bel7402 xenografts, the triangles represent the imaging data collect from SW620 xenografts and the squares represent the imaging data collect from A549 xenografts.

suggested that the FRNPs exhibited a longer duration of effective enhanced ultrasound imaging. In the blank NP groups, the mean grey value exhibited no significant difference among the 3 types of xenografts at all time points $(\mathrm{P}=0.694)$. According to the results of ultrasound imaging in vivo, the images from the FR overexpressing tumors (Bel7402 tumor xenografts and SW620 tumor xenografts) displayed a higher increased intensity value than the images from the low expressing tumors (A549 xenografts). However, we did not find similar situations such as these after the injection of blank NPs and Sonovue. These results revealed that the FRNPs achieved specific enhanced imaging for FR overexpressing tumors and the effects of tumor imaging were significantly superior to those of Sonovue at $40 \mathrm{~min}$ postinjection.

Targeting ability and biodistribution of FRNPs in vivo. The fluorescence images of Cy7-labeled FRNPs were obtained using the Kodak In-vivo Imaging System FX PRO at different time points following injection with Cy7-labeled FRNPs. The Cy7 fluorescence dye, which has absorption and emission maxima around 747 and $774 \mathrm{~nm}$, belongs to the class of NIR fluorescence dyes. NIR fluorescence imaging has many advantages, including relatively minimal autofluorescence, low tissue absorption and scatter, which results in greater tissue penetration than visible optical fluorescent dyes for in vivo imaging applications within the 650-900 nm NIR window $(29,30)$. Thus, the fluorescent images of Cy7-labeled nanoparticles can determine the in vivo biodistribution and tumor selective effect of nanoparticles (31-34).

The fluorescence imaging of xenografts were as shown in Fig. 7A, indicating the distribution of FRNPs and its aggregation in tumor region. At 1 and 5 min after the intravenous injection of the fluorescent particles, the images of xenografts were covered with different fluorescent signal strength and there were no differences in fluorescence intensity within tumor regions among the 3 types of xenografts. The fluorescence intensity of the tumor regions in the Bel7402 and SW620 tumor xenografts increased significantly from $30 \mathrm{~min}$ to $2 \mathrm{~h}$ post-injection, and high levels of fluorescence intensity remained until $12 \mathrm{~h}$. By contrast, less tumor fluorescence accumulation was observed in the A549 xenografts at the same time points. The fluorescence intensity of the tumors and muscles was detected at the different time points in order to semi-quantitatively analyze the targeting ability of the FRNPs to the FR overexpressing tumor tissues. The average of TBR between the tumor and adjacent normal thigh muscle is shown in Fig. 7B. 

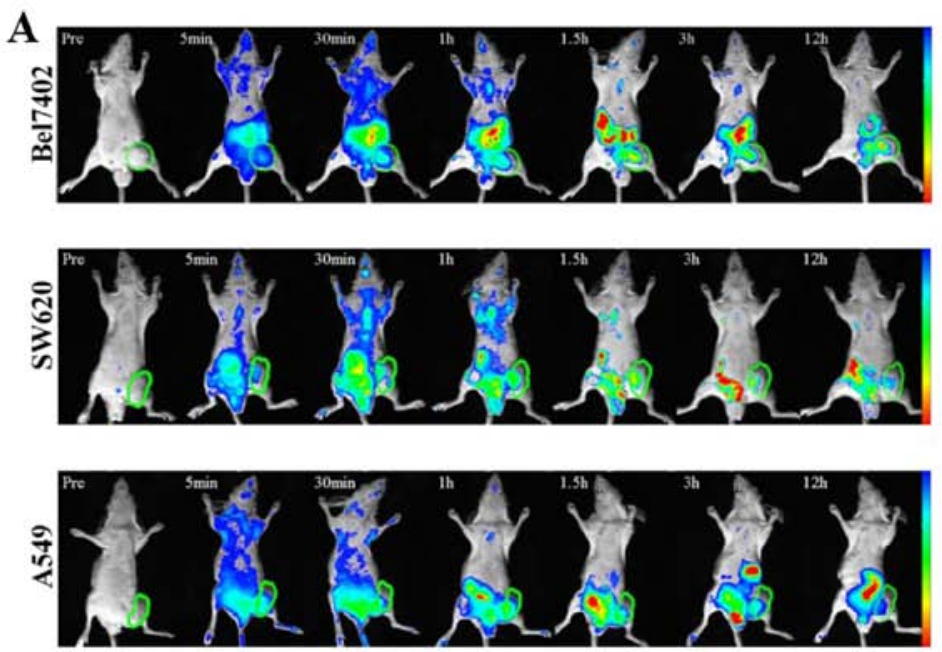

B

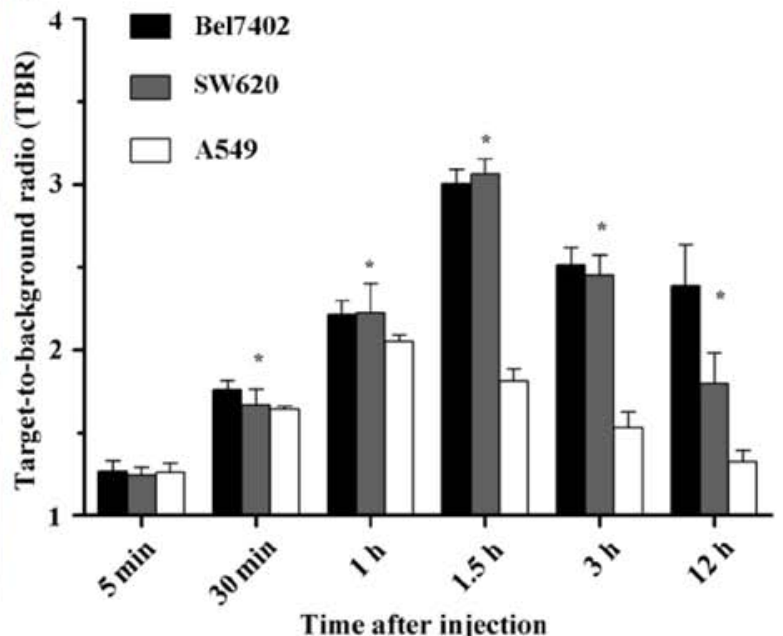

Figure 7. In vivo NIR imaging of xenografts at different time intervals after administration of Cy7-labeled FRNP and the green circles indicate the tumor regions (A). (B) Target-to-background ratio (TBR) of the xenografts after injection is shown as the mean $\pm \mathrm{SD}\left(\mathrm{n}=3\right.$ ), ${ }^{*} \mathrm{P}<0.05$. The range from blue to red in $(\mathrm{A})$ represents the changes in fluorescence intensity from weak to strong.

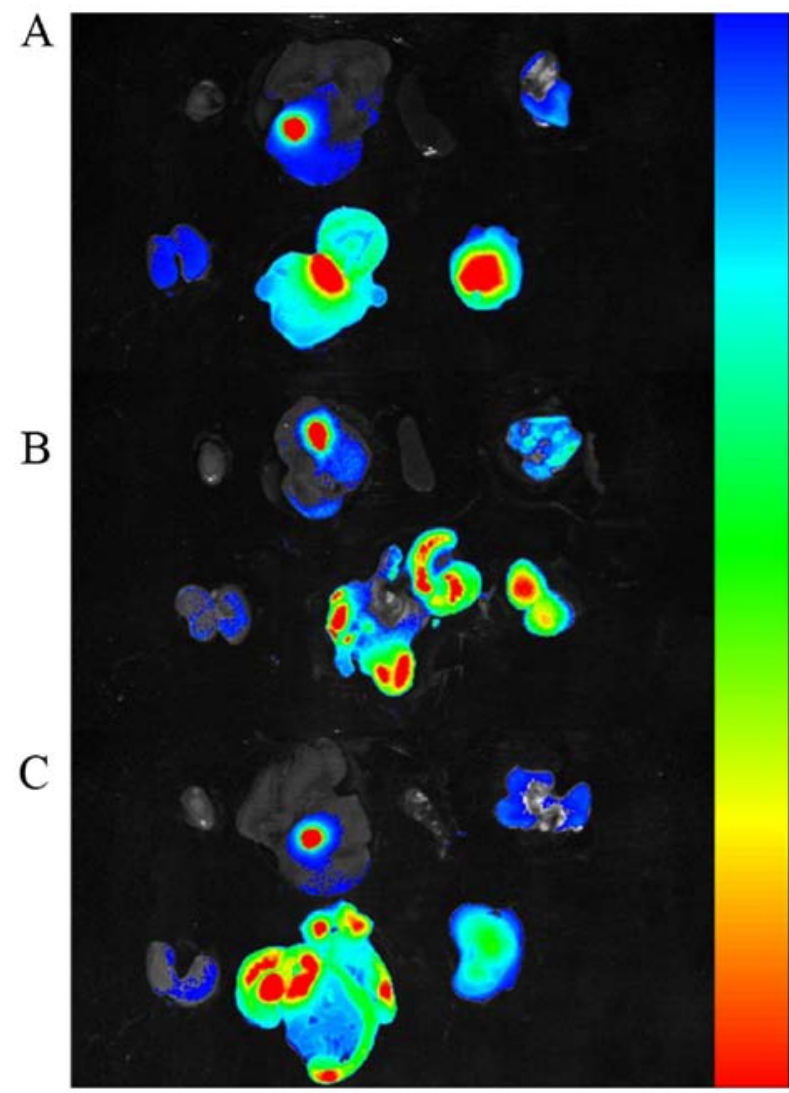

Figure 8. Ex vivo NIR imaging of other organs and tumor tissues at $12 \mathrm{~h}$ post-injection, collected from 3 types of xenografts, (A) Be17402, (B) SW620, (C) A549 tumor xenografts. (A, B and C) Organs and tumor tissue are included in proper sequence in the top row and bottom row. Top row, heart, liver, spleen, lung were arranged from left to right; bottom row, kidneys, intestines, tumor tissue were arranged from left to right. The range from blue to red represents the changes in fluorescence intensity from weak to strong.

To further determine the fluorescent signals in different tissues, the mice were sacrificed at $12 \mathrm{~h}$ post-injection and the heart, liver, spleen, lung, kidneys, intestines and tumors were excised, washed with PBS and exposed to fluorescence imaging. As shown in Fig. 8 for the ex vivo fluorescence imaging of different tissues, the fluorescent signal in the tumors of the Bel7402 and SW620 tumor xenografts was very strong, whereas little signal intensity was visible in the tumors of the A549 tumor xenografts. To semi-quantitatively analyze the targeting ability of the FRNPs, the fluorescence intensity in the tumors was measured. The average fluorescent signal intensity of the tumors excised from the Bel7402, SW620 and A549 tumor xenografts was $(2.4 \pm 0.1) \times 10^{10},(2.3 \pm 0.02) \times 10^{10},(1.24 \pm 0.03) \times 10^{10}$ photons $/ \mathrm{sec} /$ $\mathrm{mm}^{2}$ (the fluorescence images of tumors can be seen on the right side in the bottom row of Fig. 8A, B and C). The differences of fluorescent signal intensity among the 3 types of xenografts were statistically significant $(\mathrm{P}=0.027)$. These results were consistent with the outcomes from the in vivo ultrasound imaging. The ex vivo tumor imaging results also demonstrated that the FRNPs exhibited relatively high tumor-targeted distribution in the FR overexpressing tumors.

Histological analysis. To further verify the fluorescence imaging results and confirm the presence of FRNPs in tumor tissues, the frozen tissue slices of the tumor tissues were collected and fixed at 90 min post-injection of Cy7-labeled FRNPs (Fig. 9). Only a weak fluorescent signal, corresponding to autofluorescence was observed in the tumor slices of the control group. In the targeted group, we observed that the fluorescent signals of high intensity (red spots) were converged on some areas that seemed like the blood vessels of tumor tissues, whereas fluorescent signals of extravascular areas remained weak in the slices of A549 xenografts. By contrast, more and brighter fluorescent signals of extravascular areas in the slices of the Bel7402 xenografts and SW620 xenografts were observed. These results revealed that the FRNPs passed through the endothelial gap of the tumor blood vessel and combined with the tumor tissues. The targeting ability to FR overexpressing tumors and PEGylated surface against the 'cleaning up' by the reticuloendothelial system (RES) led to the accumulation of 

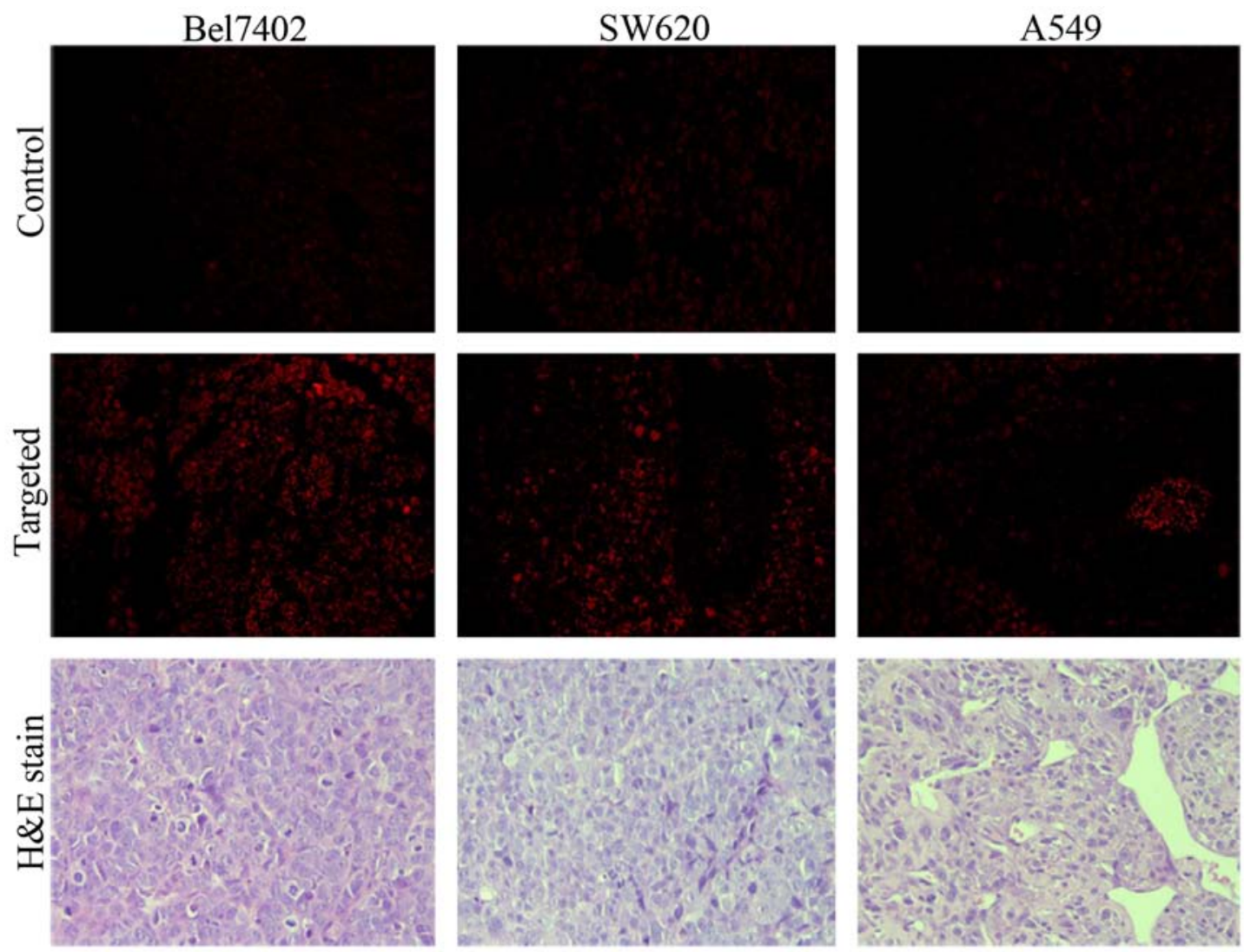

Figure 9. Fluorescent microscopy images of tumor slices from the xenografts of target and control groups after intravenous injection of folate-receptor targeted nanoparticles (FRNPs) dyed with Cy7 and physiological saline, which observed with 40X objective and x10 ocular lens. In the targeted group brighter red fluorescent spots, which corresponded to FRNPs dyed with Cy7 in the tumor tissue, were observed with a long-pass 515-560 nm emission filter cube under lamp illumination. The images of H\&E-stained tumor slices were observed under an optical microscope.

FRNPs in FR overexpressing tumors. The H\&E-stained slices of tumor tissue were observed and typical pathological mitotic figures were evident and did not exhibit any significant differences between the 2 groups.

\section{Discussion}

With the development of contrast agents, contrast-enhanced ultrasonography has played on important role in the detection and differentiation of tumors. Although many types of ultrasound contrast agents have been developed for clinical application, some clinical studies have indicated that the contrast agents currently used are limited by insufficient sensitivity and specificity $(1,4,35)$.

Nowadays, the emerging of molecular imaging introduces a new avenue for tumor detection at the molecular level with ultrasonography. However the application of molecular ultrasound imaging is required to develop nanosized contrast agents, of which the particle size is smaller than the contrast agents recently used $(7,36)$.

Currently, PFOB nanoparticles have been reported to be applied to molecular ultrasound imaging in vivo, including several molecular targeted medicine applications (37-40). The application of PFOB nanoparticles into the field of ultrasonography has been limited to low-intensity of ultrasound reflection due to nanosized particle size and the nanoparticles may also be less liable to non-specific signal enhancement events since larger concentrations (more binding events) are required to produce conspicuity $(41,42)$.

Furthermore, FR is highly expressed in various human malignancies and it has been served as a potential targeted ligand for tumor imaging and antitumor therapy due to the high affinity to bind with FA. Thus, in this study, we conducted a constructive attempt to apply the polymers of FA-CS and PEG-ALG to the preparation of the FR overexpressed tumor targeted nanoparticles (FRNPs) by using the LbL assembly technique, which maintained the advantages of FR-targeting affinity and resistance against the macrophage of RES due to PEG modification.

In this study, the targeted shell consisted of FA-CS and PEG-ALG, which were easily obtained by the carbodiimide method and did not need complex modifications. We introduced the LbLassembly techniquet the process of core-shellenveloping, which improved the quality and effectiveness of targeted shell. As shown in Fig. 2A, the particle size decreased from 244.7 \pm 6.0 to $217.6 \pm 5.6 \mathrm{~nm}$ and the surface potential was reversed (from $39.1 \pm 0.4$ to $-21.6 \pm 1.3$ ) after the first addition of PEG-ALG (layer 2). This indicated that a mesh shell on the surface of PFOB liposome was forming by the strong ionic electrostatic interaction between PEG-ALG and FA-CS. In the TEM images (Fig. 2D), we could see that FRNPs exhibited a rough surface and retained larger particle size, whereas the blank NPs (PFOB liposomes) main- 
tained a smooth surface and a small size. Although the different layers could not distinguished clearly, a darker phosphotungstically acid-stained core and a lesser-stained shell were visible. After the LbL assembly was completed, the FRNPs maintained FR-targeting function (FA modified) and solvent stability (the electrostatic repulsive force and the PEGyalted polymers that further extend the repulsive force and prevent aggregation). In the assembly process, we made an attempt to keep the particle size in an optimal range by careful adjustment of the polymer radios.

In the targeted binding assay in vitro, the FRNPs were specifically taken up by the Bel7402 and SW620 cancer cells (FR overexpressing cancer cell lines). Subsequently, we investigated the ultrasongraphy for xenograft models and found that the FRNPs obtained specific ultrasound enhanced imaging for FR overexpressing tumors. Although the enhancement of FRNPs was slightly weaker than that of Sonovue, it maintained a longer enhanced duration in FR overexpressing tumors. Furthermore, in the fluorescence imaging of the xenograft models, the ultrasound imaging results of the FRNPs were confirmed. As shown in Fig. 7, that fluorescence intensity in the FR overexpressing tumor region was significantly stronger at $30 \mathrm{~min}$ post-injection, and the ex vivo fluorescence imaging of tumors and other organs (Fig. 8) revealed that the intestines and tumors possessed a higher intensity of fluorescent signal. These results indicated that FRNPs could accumulate in FR overexpressed tumor and passed out through the endothelial gap of tumor blood vessel for targeting to FR overexpressed tumors.

In conclusion, in this study, we developed a novel strategy of ultrasonic nanoparticles modified with FA and PEG for FR overexpressingtumor imaging. FRNPs were successfully synthesized with the LbL assembly technique and maintained suitable physicochemical properties. Our data illustrated that FRNPs exhibited a respectable ability to target the certain tumors in vitro and in vivo. At present, there are certain deficiencies and limits in tumor-targeted imaging. FRNPs may thus have potential for early diagnosis and targeted therapy of FR overexpressing tumors. The nanoparticles and these methods of preparation may prove to be useful for further medical and biological applications.

\section{Acknowledgements}

The present study was supported by grants from the Social Development Major Project in Ningbo, China (no. 2012C5013).

\section{References}

1. Perera RH, Hernandez C, Zhou H, Kota P, Burke A and Exner AA: Ultrasound imaging beyond the vasculature with new generation contrast agents. Wiley Interdiscip Rev Nanomed Nanobiotechnol 7: 593-608, 2015.

2. Casciaro S, Soloperto G, Greco A, Casciaro E, Franchini R and Conversano F: Effectiveness of functionalized nanosystems formultimodal molecular sensing and imaging in medicine. IEEE Sens J 6: 2305-2312, 2013.

3. Maeda H, Wu J, Sawa T, Matsumura Y and Hori K: Tumor vascular permeability and the EPR effect in macromolecular therapeutics: A review. J Control Release 65: 271-284, 2000.

4. Hahn MA, Singh AK, Sharma P, Brown SC and Moudgil BM: Nanoparticles as contrast agents for in-vivo bioimaging: Current status and future perspectives. Anal Bioanal Chem 399: 3-27, 2011.

5. Kobayashi H, Turkbey B, Watanabe R and Choyke PL: Cancer drug delivery: Considerations in the rational design of nanosized bioconjugates. Bioconjug Chem 25: 2093-2100, 2014.
6. Orocio-Rodríguez E, Ferro-Flores G, Santos-Cuevas CL, Ramírez FM, Ocampo-García BE, Azorín-Vega E and Sánchez-García FM: Two novel nanosized radiolabeled analogues of somatostatin for neuroendocrine tumor imaging. J Nanosci Nanotechnol 15: 4159-4169, 2015.

7. Toy R, Bauer L, Hoimes C, Ghaghada KB and Karathanasis E: Targeted nanotechnology for cancer imaging. Adv Drug Deliv Rev 76: 79-97, 2014.

8. Wilson KE, Wang TY and Willmann JK: Acoustic and photoacoustic molecular imaging of cancer. J Nucl Med 54: 1851-1854, 2013.

9. Gessner R and Dayton PA: Advances in molecular imaging with ultrasound. Mol Imaging 9: 117-127, 2010.

10. Zhao R, Matherly LH and Goldman ID: Membrane transporters and folate homeostasis: Intestinal absorption and transport into systemic compartments and tissues. Expert Rev Mol Med 11: e4, 2009.

11. Shen F, Wu M, Ross JF, Miller D and Ratnam M: Folate receptor type gamma is primarily a secretory protein due to lack of an efficient signal for glycosylphosphatidylinositol modification: Protein characterization and cell type specificity. Biochemistry 34: 5660-5665, 1995.

12. Kane MA: The role of folates in squamous cell carcinoma of the head and neck. Cancer Detect Prev 29: 46-53, 2005.

13. Hartmann LC, Keeney GL, Lingle WL, Christianson TJ, Varghese B, Hillman D, Oberg AL and Low PS: Folate receptor overexpression is associated with poor outcome in breast cancer. Int J Cancer 121: 938-942, 2007.

14. O'Shannessy DJ, Yu G, Smale R, Fu YS, Singhal S, Thiel RP, Somers EB and Vachani A: Folate receptor alpha expression in lung cancer: Diagnostic and prognostic significance. Oncotarget 3: 414-425, 2012.

15. Parker N, Turk MJ, Westrick E, Lewis JD, Low PS and Leamon CP: Folate receptor expression in carcinomas and normal tissues determined by a quantitative radioligand binding assay. Anal Biochem 338: 284-293, 2005.

16. Assaraf YG, Leamon CP and Reddy JA: The folate receptor as a rational therapeutic target for personalized cancer treatment. Drug Resist Updat 17: 89-95, 2014.

17. Wu M, Gunning W and Ratnam M: Expression of folate receptor type $\alpha$ in relation to cell type, malignancy, and differentiation in ovary, uterus, and cervix. Cancer Epidemiol Biomarkers Prev 8: 775-782, 1999.

18. Kalli KR, Oberg AL, Keeney GL, Christianson TJ, Low PS, Knutson KL and Hartmann LC: Folate receptor alpha as a tumor target in epithelial ovarian cancer. Gynecol Oncol 108: 619-626, 2008.

19. Bueno R, Appasani K, Mercer H, Lester S and Sugarbaker D: The $\alpha$ folate receptor is highly activated in malignant pleural mesothelioma. J Thorac Cardiovasc Surg 121: 225-233, 2001.

20. Lu Y, Sega E and Low PS: Folate receptor-targeted immunotherapy: Induction of humoral and cellular immunity against hapten-decorated cancer cells. Int J Cancer 116: 710-719, 2005.

21. Siafaka P, Betsiou M, Tsolou A, Angelou E, Agianian B, Koffa M, Chaitidou S, Karavas E, Avgoustakis K and Bikiaris D: Synthesis of folate-pegylated polyester nanoparticles encapsulating ixabepilone for targeting folate receptor overexpressing breast cancer cells. J Mater Sci Mater Med 26: 275, 2015.

22. Liu X, Zhao J, Guo D, Wang Z, Song W, Chen W and Zhou J: Synthesis and evaluation of perfluorooctylbromide nanoparticles modified with a folate receptor for targeting ovarian cancer: in vitro and in vivo experiments. Int J Clin Exp Med 8: 10122-10131, 2015.

23. Zhou J, Romero G, Rojas E, Ma L, Moya S and Gao C: Layer by layer chitosan/alginate coatings on poly(lactide-co-glycolide) nanoparticles for antifouling protection and Folic acid binding to achieve selective cell targeting. J Colloid Interface Sci 345: 241-247, 2010.

24. Elhissi AM, O'Neill MA, Roberts SA and Taylor KM: A calorimetric study of dimyristoylphosphatidylcholine phase transitions and steroid-liposome interactions for liposomes prepared by thin film and proliposome methods. Int J Pharm 320: 124-130, 2006.

25. Salem HF, Ahmed SM, Hassaballah AE and Omar MM: Targeting brain cells with glutathione-modulated nanoliposomes: In vitro and in vivo study. Drug Des Devel Ther 9: 3705-3727, 2015.

26. Barnett BP, Ruiz-Cabello J, Hota P, Ouwerkerk R, Shamblott MJ, Lauzon C, Walczak P, Gilson WD, Chacko VP, Kraitchman DL, et al: Use of perfluorocarbon nanoparticles for non-invasive multimodal cell tracking of human pancreatic islets. Biomaterials 35 : 9984-9994, 2014. 
27. Kim J, Lee CM, Jeong HJ and Lee KY: In vivo tumor accumulation of nanoparticles formed by ionic interaction of glycol chitosan and fatty acid ethyl ester. J Nanosci Nanotechnol 11: 1160-1166, 2011

28. Alencar H, Funovics MA, Figueiredo J, Sawaya H, Weissleder R and Mahmood U: Colonic adenocarcinomas: Near-infrared microcatheter imaging of smart probes for early detection - study in mice. Radiology 244: 232-238, 2007.

29. Hawrysz DJ and Sevick-Muraca EM: Developments toward diagnostic breast cancer imaging using near-infrared optical measurements and fluorescent contrast agents. Neoplasia 2: 388-417, 2000.

30. Zheng C, Zheng M, Gong P, Jia D, Zhang P, Shi B, Sheng Z $\mathrm{Ma}$ Y and Cai L: Indocyanine green-loaded biodegradable tumor targeting nanoprobes for in vitro and in vivo imaging. Biomaterials 33: 5603-5609, 2012.

31. Hama Y, Koyama Y, Choyke PL and Kobayashi H: Two-color in vivo dynamic contrast-enhanced pharmacokinetic imaging. J Biomed Opt 12: 034016, 2007.

32. Nakamura T, Kawano K, Shiraishi K, Yokoyama M and Maitani Y: Folate-targeted gadolinium-lipid-based nanoparticles as a bimodal contrast agent for tumor fluorescent and magnetic resonance imaging. Biol Pharm Bull 37: 521-527, 2014.

33. Chung EJ, Mlinar LB, Sugimoto MJ, Nord K, Roman BB and Tirrell M: In vivo biodistribution and clearance of peptide amphiphile micelles. Nanomedicine 11: 479-487, 2015.

34. Chung EJ, Cheng Y, Morshed R, Nord K, Han Y, Wegscheid ML, Auffinger B, Wainwright DA, Lesniak MS and Tirrell MV: Fibrin-binding, peptide amphiphile micelles for targeting glioblastoma. Biomaterials 35: 1249-1256, 2014.

35. Blomqvist L, Carlsson S, Gjertsson P, Heintz E, Hultcrantz M, Mejare I and Andrén O: Limited evidence for the use of imaging to detect prostate cancer: A systematic review. Eur J Radiol 83: 1601-1606, 2014
36. Sivasubramanian M,Hsia Y and Lo LW: Nanoparticle-facilitated functional and molecular imaging for the early detection of cancer. Front Mol Biosci 1: 15, 2014.

37. Giraudeau C, Geffroy F, Mériaux S, Boumezbeur F, Robert P, Port M, Robic C, Le Bihan D, Lethimonnier F and Valette J: 19F molecular MR imaging for detection of brain tumor angiogenesis: In vivo validation using targeted PFOB nanoparticles. Angiogenesis 16: 171-179, 2013.

38. Vu-Quang H, Vinding MS, Xia D, Nielsen T, Ullisch MG, Dong M, Nielsen NC and Kjems J: Chitosan-coated poly(lactic-co-glycolic acid) perfluorooctyl bromide nanoparticles for cell labeling in (19)F magnetic resonance imaging. Carbohydr Polym 136: 936-944, 2016.

39. Watanabe T, Kimura Y and Ono T: Microfluidic fabrication of monodisperse polylactide microcapsules with tunable structures through rapid precipitation. Langmuir 29: 14082-14088, 2013.

40. Decato S, Bemis T, Madsen E and Mecozzi S: Synthesis and characterization of perfluoro-tert-butyl semifluorinated amphiphilic polymers and their potential application in hydrophobic drug delivery. Polym Chem 5: 6461-6471, 2014.

41. Marsh JN, Hall CS,Scott MJ,Fuhrhop RW, Gaffney PJ, Wickline SA and Lanza GM: Improvements in the ultrasonic contrast of targeted perfluorocarbon nanoparticles using an acoustic transmission line model. IEEE Trans Ultrason Ferroelectr Freq Control 49: 29-38, 2002.

42. Marsh JN, Partlow KC, Abendschein DR, Scott MJ, Lanza GM and Wickline SA: Molecular imaging with targeted perfluorocarbon nanoparticles: Quantification of the concentration dependence of contrast enhancement for binding to sparse cellular epitopes. Ultrasound Med Biol 33: 950-958, 2007. 